\title{
Current issue of hydrotechnical structures calculation
}

\author{
Yuri Skolubovic ${ }^{1 *}$, Yuri Bik $^{2}$, Mikhail Soppa ${ }^{1}$, Vladimir Molodin ${ }^{1}$, and Arkady Yanenko ${ }^{1}$ \\ ${ }^{1}$ Novosibirsk State University of Architecture and Civil Engineering (Sibstrin), 113, Leningradskaya, \\ Novosibirsk, 630008, Russian Federation \\ ${ }^{2}$ Siberian State University of Water Transport, 33, Schetinkina, 630000 Novosibirsk, Russia
}

\begin{abstract}
Methods of calculating hydraulic structures with the aim of identifying the reserves of their bearing capacity, extending their service life and strengthening structures at minimal cost are considered in the article. The above problems' solutions are based on the stress-strain state's study of port hydraulic structures in order to ensure the failure-free operation of facilities with the maximum reduction in time and money for their technical operation
\end{abstract}

\section{Introduction}

Operation analysis dock embankments showed that the standard service life of the docks did not correspond to the real ones, since the design of structures had not fully taken into account the intensity of physical and moral deterioration of the structures. In this regard, it became necessary to assess the reliability of the structure, during the dock embankments' examining. Docks with low level of operational reliability value must be reconstructed [1].

In order to assess the reliability of structures using the probability theory methods, it is assumed that the structure can either be in a failure state $(V)$ or in a failure-free state $(\bar{V})$, the corresponding probabilities can be calculated for each state. Since the $\bar{V}$ event is the opposite of $V$ event, then

$$
P_{f}+P_{s}=1 .
$$

As a rule, the probability of failure is taken as a measure of reliability rather than failure-free operation in construction. There are two approaches to determine the probability of failure [2,3].

The probability of failure can be estimated, based on a statistical analysis of damage. However, this method implementation in determining the reliability of dock facilities is problematic, since dock walls are structures with great reliability and their failure is a rare event with a very low probability.

Consequently, a statistical estimation of the failure probability would require such a large sample size, which is almost impossible to achieve, since there are only a small number of completely similar dock embankments. In addition, even if it is possible to draw

\footnotetext{
* Corresponding author: author@email.org
} 
any reliable conclusions based on damage statistics, these conclusions will appear, having historical interest only. Thus, it is hardly possible to estimate the probability of failure from statistics.

Nevertheless, it is possible to determine characteristics of a mass nature and evaluated statistically on the basis of random values for individual structures, such as dock embankments. These characteristics include properties of building materials, produced and tested as mass products, repeated external loads, geometric dimensions, physical and mechanical characteristics of soils, joints elements. All these parameters in the theory of structural reliability can be considered as basic variables determined by statistical studies. Mathematically, they are represented as random variables and are denoted by $X_{1}, X_{2}, \ldots$, $X_{m}$, one can theoretically determine the probability of failure conditions occurrence, based on the introduced variables [4].

It must be borne in mind that such defined reliability of dock facilities, naturally, does not take into account the possible negative contribution due to erroneous human actions.

So, it is possible to reliably estimate the middle part of distribution density curve, and accordingly the mean and standard deviations with the help of statistical studies of such durable structures operation as dock walls, but it is almost impossible to justify the behavior in the low distribution densities zones.

Therefore, in the future, we will use the concept of "operational failure probability" to justify the dock embankments reliability, which can only be considered as a comparative value for a qualitative assessment of the structures' strength and reliability $[5,6]$.

\section{Materials and methods}

Real building structures are made of materials which properties depend on time and impact of various environmental factors. All materials are subjected to aging processes, fatigue, hardening, corrosion, creep and other phenomena that change bearing capacity, most often in the reduction direction. These phenomena significantly affect the probability of failure.

Therefore, it is clear that the probability of failure increases monotonically over the time. If we assume that the structure at the initial moment of its existence $(t=0)$ is in a state of trouble-free operation, then there are two boundary values:

$$
P_{f}(0)=0 \text { and } \lim _{t \rightarrow \infty} P_{f}(t)=1 \text {. }
$$

The reliability characteristic of a structure over the time is the failure density $h_{t}(t)$,, which allows determining the probability of failure in the time interval $(t, t+\Delta t)$ for a structure that has already reached the age $t$. The value of $h_{t}(t) \Delta t$ is calculated as the probability that the structure works without fail until time $t$ and can fail, at least, in the following time interval $(t, t+\Delta t)$ :

$$
h_{t} \Delta t=P\left(T_{L} \leq t+\Delta t \mid T_{L}>t\right) .
$$

where $T_{L}$ is the lifetime of the structure.

The failure density, presented graphically, almost always has the form, which is shown in Figure 1. At the beginning of the existence period (phase 1), there are failure cases of any elements due to the poor quality of work and materials quite often. This is followed by phase 2 with a relatively constant density of failures, which are usually caused by overloads. Due to the processes of fatigue, aging, or corrosion, the failure density increases again at the end of the building's service life (phase 3).

It must be borne in mind that failure can not follow only due to a single crossing of the critical level, assessing the possibility of a structure failure as a whole. The occurring 
phenomenon of material fatigue can lead to sudden fatigue failure at a relatively low load level within frequently repeated loads.

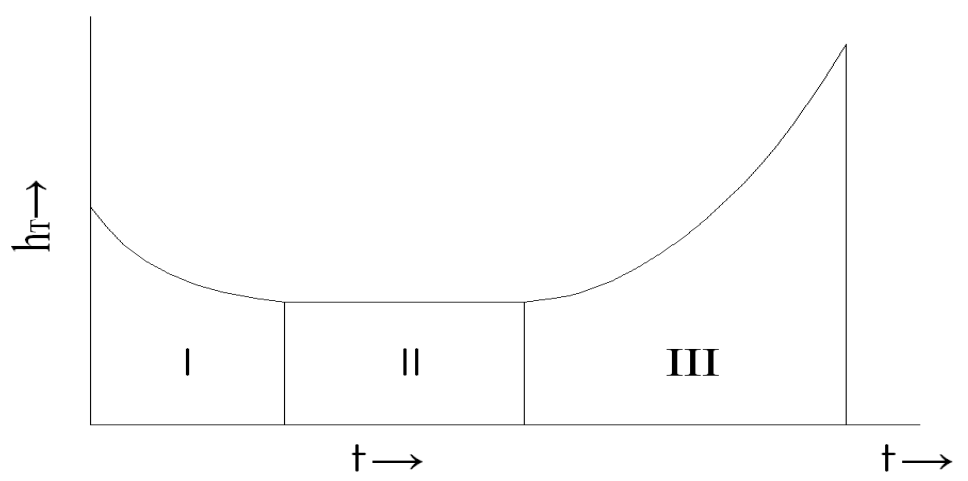

Fig. 1. Failure Density Graph

Thus, one of the main tasks of the reliability theory is to evaluate reliability and durability indicators of a system as a whole according to the known laws of reliability and durability distribution of its elements. The method of calculating reliability and durability indicators substantially depends on how the structural elements interact with each other in terms of ensuring system reliability.

In order to assess the reliability of the dock embankments, it is necessary to use the dependence to determine the probability of a structure failure as a whole. One must first find the reliability indicators of each element.

The failure-free operation probability of dock embankments is determined by the calculation formula that is valid for the series connection of system elements where, at least one failure leads to the entire system failure as a whole:

$$
P_{s}=\prod_{k=1}^{m} P_{s k} \text {. }
$$

Here $m$-is the number of elements with a probability of failure-free operation $P_{\mathrm{s} 1}, P_{s 2}$, $\ldots, P_{s \mathrm{~m}}$. In this case, the behavior of structural elements is considered as a random process, and the limiting state is in the form of a random ejection from the region of admissible states.

Thus, in order to assess the element's reliability, the question of whether the random process reaches a certain acceptable level or exceeds it plays an important role.

In the case when the random variables have a normal distribution, and the equation of limit state is linear with respect to $X_{i}$, the reliability of the structure can be estimated by the approximate method. With a normal distribution of random variables, the distribution density is expressed as:

$$
f_{x}\left(x_{1}, x_{2}, \ldots, x_{m}\right)=\left[\frac{1}{(2 \pi)^{m / 2}} \prod_{i=1}^{m} \sigma_{x_{i}}\right] \exp \left\{-0,5 \sum_{i=1}^{m}\left[\left(x_{i}-m_{x i}\right) / \sigma_{x i}\right]^{2}\right\}
$$

If the equation of limit state is linear, then it can be written as

$$
g(x)=c_{0}+\sum_{i=1}^{m} c_{i} x_{i}
$$

where $c_{i}$ are constants, determined by the structure of the static system.

Further calculations are greatly simplified after the variables replacement 


$$
y_{i}=x_{i}-\frac{m_{x i}}{\sigma_{x i}} .
$$

The new variable has zero mean and unit standard deviation. Then the distribution density of the new quantity is

$$
f_{y}\left(y_{1}, y_{2}, \ldots, y_{m}\right)=\left[\frac{1}{(2 \pi)^{0,5 m}}\right]\left(-0,5 \sum_{i=1}^{m} y_{i}^{2}\right) .
$$

The limit state function in $y$ - space can be written as

$$
h(y)=c_{0}+\sum_{i=1}^{m} c_{i} m_{x i}+\sum_{i=1}^{m} c_{i} \sigma_{x i} y_{i}=0 .
$$

The equation of limiting state in $y$-space is also linear; it is transformed into the Hessian form

$$
h(y)=\sum_{i=1}^{m} \alpha_{i} y+\beta=0
$$

where $\alpha_{i}$ is the sensitivity coefficient

$$
\alpha_{i}=\frac{c_{i} \sigma_{x i}}{\sqrt{\sum_{i=1}^{m}\left(c_{i} \sigma_{x i}\right)^{2}}} ;
$$

$\beta$ is safety index

$$
\beta=c_{0}+\frac{\sum_{i=1}^{m} c_{i} m_{x i}}{\sqrt{\sum_{i=1}^{m}\left(c_{i} \sigma_{x i}\right)^{2}}} .
$$

Dependence probability

$$
P_{f}=\int_{V} \ldots \int \prod_{i=1}^{m} f_{x i}\left(x_{i}\right) d x_{i}
$$

It is assumed that the functions and distribution densities of all random variables $x_{i}$ are completely known in the above reasoning. However, a large volume of samples is required, especially for "peripheral" distribution zones to establish the type of distribution with sufficient reliability. As it was mentioned, the corresponding experimental points, as a rule, are not enough, studying dock embankments. At the same time, the type of distribution, determined primarily by peripheral zones, has a decisive influence on the failure probability. The method of moments allows avoiding these difficulties, whereby it is not the probability of failure that is determined, but the safety index, which does not depend on a distribution type [7].

The safety index is calculated based on the mathematical expectation $m_{x i}$ and standard deviation $\sigma_{x i}$, which are based on the available statistical information about the structure.

The moments' method determines the safety factors

$$
z=g\left(x_{1}, x_{2}, \ldots, x_{m}\right)
$$

according to the first two moments of given random variables $x_{i}$.

If the safety factor is a linear function of the basis variables

$$
z=c_{o}+\sum_{i=1}^{m} c_{i} x_{i}
$$


then the moments of the random variables' functions according to probability theory can be determined by the given mathematical expectation $E\left[x_{i}\right]=m_{x i}$, variance $\operatorname{Var}\left[x_{i}\right]=\sigma_{x i}{ }^{2}$ and covariance $\operatorname{Cov}\left[x_{i}, x_{j}\right]=\sigma_{x i} \sigma_{x j} \rho_{x i x j}$ :

$$
\begin{aligned}
& E[z]=m_{z}=\int_{-\infty}^{\infty} \ldots \int g\left(x_{1}, x_{2}, \ldots, x_{m}\right) f_{x}\left(x_{1}, x_{2}, \ldots, x_{m}\right) \prod_{i} d x_{i} \\
& \operatorname{Var}[z]=\sigma_{z}^{2}=\int_{-\infty}^{\infty} \ldots \int\left\{g\left(x_{1}, x_{2}, \ldots, x_{m}\right)-\right. \\
& \left.-E\left[g\left(x_{1}, x_{2}, \ldots, x_{m}\right)\right]\right\}^{2} f_{x}\left(x_{1}, x_{2}, \ldots, x_{m}\right) \prod_{i} d x_{i}
\end{aligned}
$$

Taking into account, that

$$
\left.\begin{array}{l}
\int_{-\infty}^{\infty} \ldots \int f_{x}\left(x_{1}, x_{2}, \ldots, x_{m}\right) \prod d x_{i}=1 \\
\int_{-\infty}^{\infty} \ldots \int x_{j} f_{x}\left(x_{1}, x_{2}, \ldots, x_{m}\right) \prod_{i} d x_{i}=E\left[x_{j}\right]=m_{x j} \\
\int_{-\infty}^{\infty} \ldots \int\left(x_{j}-m_{x j}\right) f_{x}\left(x_{1}, x_{2}, \ldots, x_{m}\right) \prod_{i} d x_{i}=0 ; \\
\int_{-\infty}^{\infty} \ldots \int\left(x_{j}-m_{x j}\right)^{2} f_{x}\left(x_{1}, \ldots, x_{m}\right) \prod_{i} d x_{i}=\operatorname{Var}\left[x_{j}\right]=\sigma_{x j}{ }^{2} \\
\int_{-\infty}^{\infty} \ldots \int\left(x_{j}-m_{x j}\right)\left(x_{k}-m_{x k}\right) f_{x}\left(x_{1}, \ldots, x_{m}\right) \prod d x_{i}=\operatorname{Cov}\left[x_{j}, x_{k}\right],
\end{array}\right\}
$$

we get

$$
\left.\begin{array}{l}
E[z]=m_{z}=c_{o}+\sum_{i=1}^{m} c_{i} m_{x i}, \\
\operatorname{Var}[z]=\sigma_{z}^{2}=\sum_{i=1}^{m} c_{i}^{2} \sigma_{x i}{ }^{2}+\sum_{i=1}^{m} \sum_{j=1}^{m} c_{i} c_{j} \operatorname{Cov}\left[x_{i}, x_{j}\right]
\end{array}\right\}
$$

or in the matrix form

$$
\left.\begin{array}{l}
E[z]=m_{z}=c_{o}+c^{T} E[x], \\
\operatorname{Var}[z]=\sigma_{z}{ }^{2}=c^{T} C_{x} c, \quad
\end{array}\right\}
$$




$$
C=\left(\begin{array}{c}
C_{1} \\
\cdot \\
\cdot \\
\cdot \\
C_{m}
\end{array}\right) ; \quad E[x]=\left(\begin{array}{c}
m_{x 1} \\
\cdot \\
\cdot \\
\cdot \\
m_{x m}
\end{array}\right) .
$$

The safety index is defined as the mathematical expectation ratio of safety margin to its standard deviation

$$
\beta_{c}=\frac{m_{z}}{\sigma_{z}}=\frac{E[z]}{\sqrt{\operatorname{Var}[z]}} .
$$

If the equation of limit state is nonlinear, it can be linearized using the Taylor expansion:

$$
g\left(x_{1}, \ldots, x_{m}\right)=g\left(m_{x 1}, \ldots, m_{x m}\right)+\sum_{i=1}^{m}\left(x_{i}-m_{x i}\right) \partial g /\left.\partial x\right|_{x=m} .
$$

So, the above expressions for the mathematical expectation and standard deviation can be in the form:

$$
\left.\begin{array}{l}
E[z]=g\left(m_{x 1}, \ldots, m_{x m}\right\} ; \\
\operatorname{Var}[z]=\sum_{i=1}^{m}\left(\partial g / \partial x_{i}\right)^{2} \operatorname{Var}\left[x_{i}\right]+\sum_{i=1}^{m} \sum_{i \neq j}^{m}\left(\frac{\partial^{2} g}{\partial x_{i} \partial x_{j}}\right) \operatorname{Cov}\left[x_{i}, x_{j}\right] .
\end{array}\right\}
$$

We have to note that all derivatives, included in the formula, are calculated at the point of mathematical expectation.

We introduce the derivatives' vector of the limit state equation at the point of mathematical expectation

$$
G(m)=\left(\begin{array}{c}
\partial g /\left.\partial x_{1}\right|_{x=m} \\
\cdot \\
\cdot \\
\partial g /\left.\partial x_{m}\right|_{x=m}
\end{array}\right) ; \quad \text { where } m=E[x] .
$$

Then the safety index, also called the Cornell index, will have the form of

$$
\beta=\frac{E[z]}{\sqrt{\operatorname{Var}[z]}}=\frac{g(m)}{\sqrt{G^{T}(m) C_{X} G(m)}} .
$$

The above-described approach to determining the structure reliability by the Cornell safety index is quite simple and at the same time gives acceptable results in accuracy. In this regard, it can be recommended for a rapid assessment of the dock embankments' state. If it is necessary to conduct more accurate calculations, it is advisable to use the method of semi-invariants, successfully tested by V. D. Kostyukov.

The half of the total length of the dock front is quays, which are made of metal sheet pile. The most likely types of failure for quays are destruction of the front wall and rupture of anchor rods, so, we will illustrate the author's recommended method for assessing the reliability of dock embankments in these two cases [8].

The strength of the quays' front wall is determined from the condition

$$
W \sigma_{T}-M \geq 0,
$$


where $W$-resistance moment of the front wall;

$\sigma_{T}$ - front wall yield strength;

$M$ - moment in the front wall.

We consider random uncorrelated quantities $W$ and $\sigma_{T}$. The momentum in the front wall is neglected. The distribution parameters of random variables can be taken under consideration, based on the authors' studies on the dock embankments of various Siberian rivers (Table 1).

Table 1. Distribution parameters for a front wall

\begin{tabular}{|c|c|c|}
\hline Random value & $m_{x i}=E\left[x_{i}\right]$ & $\sigma_{x i}=\left(\operatorname{Var}\left[x_{i}\right]\right)^{1 / 2}$ \\
\hline$x_{1}=W$ & $2,9010^{-3} \mathrm{~m}^{3}$ & $3,0010^{-4} \mathrm{~m}^{3}$ \\
\hline$x_{2}=\sigma_{T}$ & $2,8110^{8} \mathrm{~Pa}$ & $2,7010^{7} \mathrm{~Pa}$ \\
\hline
\end{tabular}

The equation of the limiting state of the front wall has the form:

$$
g(x)=W \sigma_{T}-M=x_{1} x_{2}-M=0 \text {. }
$$

The equation is nonlinear due to the fact that not only the resistance moment in the wall is random, but also the yield strength of the material. We linearize it, using the Taylor method:

$$
g(m)=m_{W} m_{\sigma T}-M
$$

We take a bending moment in the front wall $\mathrm{M}=500 \mathrm{kN} / \mathrm{m}$ then

$$
\begin{aligned}
& g(m)=2,9 \cdot 10^{-3} \cdot 2,81 \cdot 10^{8}-50000=3,15 \cdot 10^{5} ; \\
& C(x)=\left(\begin{array}{cc}
9 \cdot 10^{-8} & 0 \\
0 & 7,29 \cdot 10^{14}
\end{array}\right) \text {; } \\
& \partial g / \partial W=\sigma_{T} ; \quad \partial g /\left.\partial W\right|_{x=m}=m_{\sigma T}=2,81 \cdot 10^{8} ; \\
& \partial g / \partial \sigma_{T}=W ; \quad \partial g /\left.\partial \sigma_{T}\right|_{x=m}=m_{W}=2,9 \cdot 10^{-3} \text {; } \\
& G(m)=\left(\begin{array}{c}
2,81 \cdot 10^{8} \\
2,90 \cdot 10^{-3}
\end{array}\right) \\
& \operatorname{Var}[z]=G^{T}(m) C_{X} G(m)=\left(\frac{\partial g}{\partial W}\right)^{2}{\sigma_{W}}^{2}+\left(\frac{\partial g}{\partial \sigma_{T}}\right)^{2} \sigma_{\sigma T}{ }^{2}= \\
& =\left(2,81 \cdot 10^{8}\right)^{2}\left(3 \cdot 10^{-4}\right)^{2}+\left(2,9 \cdot 10^{-3}\right)^{2}\left(2,7 \cdot 10^{7}\right)^{2}=132,37 \cdot 10^{8} .
\end{aligned}
$$

As a result of the calculations, we obtain the safety index

$$
\beta_{C}=\frac{g(m)}{\sqrt{\operatorname{Var}[z]}}=\frac{3,15 \cdot 10^{5}}{\sqrt{132,37 \cdot 10^{8}}}=2,74
$$

In order to determine the reliability of the quays' front wall, corresponding to the found safety index, table 2 is compiled.

Table 2. Relationship between safety index and probability of uptime

\begin{tabular}{|c|c|c|c|c|c|c|}
\hline$\beta$ & 0 & 1 & 2 & 3 & 4 & 5 \\
\hline$P_{S}$ & 0,500 & 0,841 & 0,977 & 0,998 & 0,999 & 1,000 \\
\hline
\end{tabular}

The table shows that the safety index $\beta_{C}=2,74$ corresponds to the probability of failurefree operation $\mathrm{P}_{S}=0,992$. 
The result is excellently correlated with V.D. Kostyukov data, although it was obtained quickly and easily by the approximate calculation method.

The fairly simple mathematical calculations, used in the method, can be reduced with the help of nomogram, compiled by the authors of the article (Figure 2), which allows one to determine the value of $(\operatorname{Var}[z])^{1 / 2}$, i.e. the denominator in the expression for the safety index $\beta$. In the nomogram, $\sigma_{T}$ and $m_{\sigma T}$ are in $\mathrm{MPa}, \sigma_{W}$ and $m_{W}$ in $\mathrm{M}^{3}$.

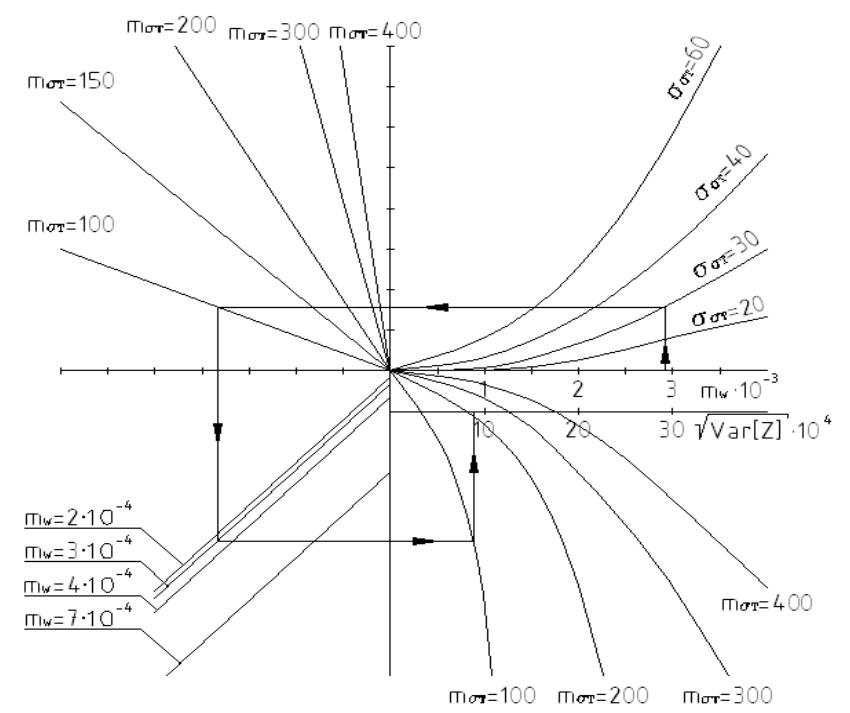

Fig. 2. Nomogram to the definition $\sqrt{\operatorname{Var}[z]}$ for quays' front wall

We determine the probability of failure-free operation of anchor rods. The cross section strength of steel anchor rods is determined from the condition

where $d$ - is rods' diameter;

$$
\pi d^{2} \sigma_{T}-6 R l \geq 0
$$

$R$ - is the anchor force per 1 linear meter of the structure;

$l$-distance between anchors.

The diameter of the anchor rod can change significantly because of corrosion. Therefore, we take $d$ and $\sigma_{T}$ as random uncorrelated values. The spread of anchor forces and the distance between the anchors is neglected [9]. The distribution parameters of random variables are presented in table 3 .

Table 3. Distribution parameters for anchor rods

\begin{tabular}{|c|c|c|}
\hline Random value & $m_{x i}=E\left[x_{i}\right]$ & $\sigma_{x i}=\left(\operatorname{Var}\left[x_{i}\right]\right)^{1 / 2}$ \\
\hline$x_{1}=d$ & $8,0010^{-2} \mathrm{~m}^{3}$ & $6,0010^{-3} \mathrm{~m}^{3}$ \\
\hline$x_{2}=\sigma_{T}$ & $2,8110^{8} \mathrm{~Pa}$ & $2,7010^{7} \mathrm{~Pa}$ \\
\hline
\end{tabular}

The equation of the limiting state of anchor rods

$$
g(x)=\pi d^{2} \sigma_{T}-6 R l=0 .
$$

We linearize the equation for the Taylor expansion:

$$
g(m)=\pi m_{d}^{2} m \sigma_{T}-6 R l=0 .
$$

We take the anchor force $R=481,5 \mathrm{kN}$, and the distance between anchors $l=1,6 \mathrm{~m}$, then 


$$
\begin{gathered}
g(m)=\pi\left(8 \cdot 10^{-2}\right)^{2} 2,81 \cdot 10^{8}-6 \cdot 481,5 \cdot 10^{3} \cdot 1,6=10,25 \cdot 10^{5} ; \\
C_{X}=\left(\begin{array}{cc}
36 \cdot 10^{-6} & 0 \\
0 & 7,29 \cdot 10^{14}
\end{array}\right) ; \\
\frac{\partial g}{\partial d}=2 \pi \sigma_{T} d ; \quad \partial g /\left.\partial d\right|_{x=m}=2 \pi m_{\sigma T} m_{d}=141,17 \cdot 10^{6} ; \\
\frac{\partial g}{\partial \sigma_{T}}=\pi d^{2} ; \quad \partial g /\left.\partial \sigma_{T}\right|_{x=m}=\pi m_{d}{ }^{2}=200,96 \cdot 10^{-4} ; \\
G(m)=\left(\begin{array}{c}
132,3 \cdot 10^{6} \\
176,6 \cdot 10^{-4}
\end{array}\right) ; \\
\operatorname{Var}[z]=G^{T}(m) C_{X} G(m)=\left(\frac{\partial g}{\partial W}\right)^{2} \sigma_{d}{ }^{2}+\left(\frac{\partial g}{\partial \sigma_{T}}\right)^{2} \sigma_{\sigma T}{ }^{2}= \\
\left(141,17 \cdot 10^{6}\right)^{2}\left(6 \cdot 10^{-3}\right)^{2}+\left(200,96 \cdot 10^{-4}\right)^{2}\left(2,7 \cdot 10^{7}\right)^{2}=10,11 \cdot 10^{11} .
\end{gathered}
$$

As a result of the calculations, we obtain the safety index

$$
\beta_{C}=\frac{g(m)}{\sqrt{\operatorname{Var}[z]}}=\frac{10,25 \cdot 10^{5}}{\sqrt{10,11 \cdot 10^{11}}}=1,02 .
$$

In accordance with table 3 , we determine the probability of failure-free operation of anchor rods $P_{S}=0,844$.

The authors compiled a nomogram for $(\operatorname{Var}[z])^{1 / 2}$ determination to facilitate the calculation of the safety index for anchor rods' operation (Figure 3). On the nomogram, $m_{\sigma T}$ and $\sigma_{\sigma T}$ in МПа, $m_{d}$ and $\sigma_{d}$ are in meters.

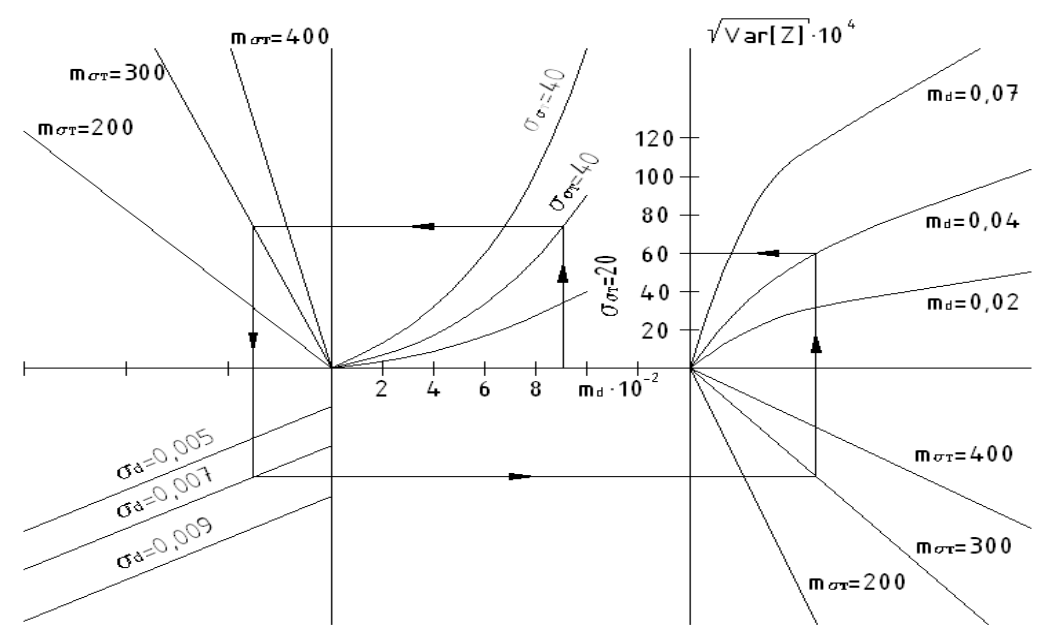

Fig. 3. Nomogram to the definition for anchor rods

\section{Results}

The methodology, proposed by the authors for determining the reliability of structural elements by the safety index, can also be used to assess failure probability of various structural elements of dock embankments [10]. 
The authors recommend a quick assessment of the reliability of existing dock facilities to use the nomograms proposed in this work, which make it possible to accurately assess the state of the studied structural elements

\section{References}

1. Yu. I. Bik, M. A. Scherbinin. Reliability assessment of hydraulic structures. Textbook for high schools. Novosibirsk: NGAVT, 122 (2010).

2. Yu.I. Bik, V.V. Degtyareva. Safety index as a measure of the reliability of building structures. Far East. 3-4, 57-59 (2016)

3. Yu.I. Bik. Experimental studies of the stress-strain state of hydraulic structures. Novosibirsk. SSUVT 105 (2018)

4. E. L. Voytov, Z. P. Nesterenko and A. G. Pchelkin. The study of biochemical processes in batches of filters tertiary sewage treatment. Izvestiya Vysshikh Uchebnykh Zavedenii. Energy 9 92-97 (2009)

5. C. F. Forster and D. A. J. Wase. Environmental biotechnology. Chichester: Ellis Horwood Ltd 453 (2011)

6. Yu.V. Voronov and S.V. Yakovlev. Wastewater and wastewater treatment Moscow: Publishing Association of Construction Universities 704 (2016)

7. V. B. Smirnov and D. A. Guskov. Highly effective granular filters for post-treatment of biologically treated sewage. Plumbing, heating, air conditioning $\mathbf{6}$ (2017)

8. E.L. Voytov and Y .L. Skolubovich Preparation of drinking water from surface sources with a high natural and anthropogenic pollution. Novosibirsk: Novosibirsk state university of architecture and civil engineering (Sibstrin) 216 (2010)

9. E.L. Voitov and Y.L. Skolubovich Pat. 2307075 RF, MKI C 02 F 1/52. (2010)

10. A.M. Blagorazumova. Treatment and dewatering of urban wastewater sludge. St. Petersburg.

Lan.

208

(2014) 